\title{
Vehicle development - how good are the forecasts? A look back and into the future
}

Prof. Dr. Peter E. Pfeffer, Automotive Engineering, Munich University of Applied Sciences;

Prof. Dr. U. Seiffert, WiTech Engineering GmbH

This manuscript is not available according to publishing restriction. Thank you for your understanding. 